\section{(6) OPEN ACCESS}

\title{
$\alpha$-Methylacyl-CoA racemase (AMACR) serves as a prognostic biomarker for the early recurrencel metastasis of HCC
}

\author{
Bo $\mathrm{Xu}_{1}^{1,2,3}$ Zhixiong Cai, ${ }^{2,3}$ Yongyi Zeng, ${ }^{1,2,3}$ Lihong Chen, ${ }^{2,3,4}$ Xiaobo $\mathrm{Du}^{5}$ \\ Aimin Huang, 2,3,4 Xiaolong Liu, 2,3 Jingfeng Liu ${ }^{1,2,3}$
}

- Additional material is published online only. To view please visit the journal online (10.1136/jclinpath-2014202378)

1 Liver Disease Center, The First Affiliated Hospital of Fujian Medical University, Fuzhou, People's Republic of China ${ }^{2}$ The United Innovation of Mengchao Hepatobiliary Technology Key Laboratory of Fujian Province, Mengchao Hepatobiliary Hospital of Fujian Medical University, Fuzhou, People's Republic of China ${ }^{3}$ The Liver Center of Fujian Province, Fujian Medical University, Fuzhou, People's Republic of China

${ }^{4}$ Department of Pathology, School of Basic Medical

Science, Fujian Medical University, Fuzhou, People's Republic of China ${ }^{5}$ Department of Urology, The First People's Hospital of Yueyang, Yueyang, People's Republic of China

\section{Correspondence to} Professor Xiaolong Liu and Professor Jingfeng Liu, Mengchao Hepatobiliary Hospital of Fujian Medical University, Xihong Road 312, Fuzhou 350025, Fujian Province, P.R. China; xiaoloong.liu@gmail.com, drjingfeng@126.com

Received 22 April 2014 Revised 1 July 2014 Accepted 19 July 2014 Published Online First 4 August 2014

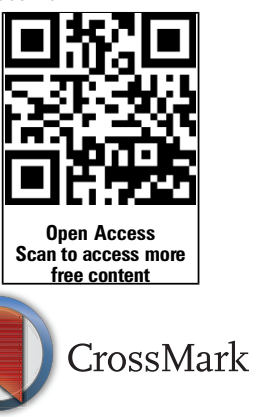

To cite: $X u B, C a i Z$ Zeng Y, et al. J Clin Pathol 2014;67:974-979.

\section{ABSTRACT}

Aims Hepatocellular carcinoma (HCC) is one of the most common malignancies worldwide, and it is still lacking effective prognostic biomarkers so far. Previous results of the iTRAQ-based quantitative proteomics study (iTRAQ-2DLC-MS/MS) have shown that $\alpha$-methylacylCoA racemase (AMACR) might be a promising prognostic biomarker for the early recurrence/metastasis of hepatocellular carcinoma (HCC). Here a large-scale cohort clinical study was performed to evaluate its prognostic potential.

Methods HCC samples from patients $(n=158)$ were used for the construction of tissue microarray. The expression level of AMACR was determined by immunohistochemical staining. A large-scale cohort clinical study between the expression of AMACR and some major clinical parameter has been performed to assess the prognostic potential of AMACR for the early recurrence/metastasis of HCC.

Results Some important clinical parameters such as $\alpha$ fetoprotein, tumour numbers, dissemination to regional lymph nodes, tumour capsule and portal vein tumour thrombosis are significantly associated with the low expression of AMACR. The expression of AMACR was an independent factor for the survival of patients with HCC. The median survival time was 17 months in the lowexpression group compared with 45 months in the highexpression group.

Conclusions This study reveals that the AMACR might be a potential prognostic marker for predicting early recurrence/metastasis of HCC after hepatectomy.

\section{INTRODUCTION}

Hepatocellular carcinoma (HCC) is one of the most common malignancies worldwide and is reported to be the second leading cause of cancer death in China. ${ }^{1}$ Despite the proved feasibility and safety of surgical resection for HCC, ${ }^{23}$ the prognosis of patients with HCC remains poor, mainly due to the intrahepatic recurrence and/or extrahepatic metastasis. In such cases, it is necessary to identify new biomarkers for distinguishing, predicting and treating the early and late recurrence/metastasis, which will certainly give important guides for identifying patients with HCC with high risk of early recurrence/metastasis.

$\alpha$-Methylacyl-CoA racemase (AMACR) is a peroxisomal and mitochondrial enzyme that plays an important role in bile acid biosynthesis and $\beta$-oxidation of branched-chain fatty acids through the interconversion of (R)- and (S)-2-methyl branched-chain fatty acyl-CoA fragments. ${ }^{4}$ It is abundantly expressed in prostate cancer compared with the benign prostate epithelium and serves as a diagnostic biomarker. ${ }^{5-8}$ There are also some reports regarding the correlations between the expression of AMACR and the diagnostic potential for HCC. Guzman et $a l^{9}$ have reported that AMACR could distinguish the HCC and the dysplastic hepatocytes from the benign nondysplastic hepatocytes based on the different staining patterns, but the sensitivity was not mentioned in this report; Li et $a l^{10}$ have reported that AMACR could serve as a useful marker for distinguishing the welldifferentiated HCC from the hepatocellular adenoma, and the AMACR might play a role in HCC development and progression. However, there has been conflicting reports in other studies where it was found that AMACR may not be a suitable marker; Willemoe et al ${ }^{11}$ have reported that the staining patterns and staining intensities of AMACR in HCC tumour cells were not significantly different from the surrounding none tumour cells, and the AMACR staining could not distinguish the neoplastic from non-neoplastic liver cells. These reports make the HCC diagnostic potential of AMACR inconclusive, and it needs further large-scale study and elucidation.

In our previous quantitative proteomics study (iTRAQ-2DLC-MS/MS), ${ }^{12}$ the results have shown that AMACR might be a very promising prognostic biomarker for the early recurrence/metastasis of HCC instead of a diagnostic biomarker. Here, a large-scale cohort clinical study between the expression of AMACR and some major clinical parameter has been performed to assess the prognostic potential of AMACR for predicting the early recurrence/ metastasis of HCC.

\section{MATERIALS AND METHODS \\ Sample collection}

One hundred and fifty-eight formalin-fixed and paraffin-embedded HCC tissues from consecutive patients who underwent curative resection between 2002 and 2010 at the First Affiliated Hospital of Fujian Medical University were retrieved for immunohistochemical staining. Barcelona Clinic Liver Cancer (BCLC) of the evaluated patients were $0(6$, $3.8 \%)$, A $(22,13.9 \%)$, B $(130,82.3 \%)$. The tissues were divided into three groups according to the time of recurrence/metastasis after operation: the patients who had recurrence/metastasis within 12 months after operation $\left(\mathrm{R} / \mathrm{M}_{\leq 12}\right.$ months group, $\mathrm{n}=101$ ), and the BCLC of the evaluated patients 
were A (7), B (94); the patients whose recurrence/metastasis occurred between 12 and 24 months after operation $\left(\mathrm{R} / \mathrm{M}_{12}\right.$ 24 months group, $\mathrm{n}=21$ ), and the BCLC of the evaluated patients were A (5), B (16); the patients who had no recurrence/metastasis at least 24 months after surgery $(\mathrm{NR} / \mathrm{M}$ group, $\mathrm{n}=36)$, and the BCLC of the evaluated patients were 0 (6), A (10), B (20). Fresh tissues were collected at the time of surgery from patients and were formalin embedded for immunohistochemistry. The project was approved for the using of human biopsy by the Institution Review Board of the First Affiliated Hospital of Fujian Medical University. The written consent was received from all participants before surgery. Clinical and pathological diagnosis of patients with HCC met the diagnostic criteria of the American Association for the Study of Liver Diseases.

\section{Construction of the tissue microarrays and immunohistochemistry}

HCC samples from patients $(n=158)$ were used for the construction of tissue microarray (TMA). By using manual tissue arrayer, tissue cores from circled areas were targeted and then transferred to the corresponding location of the recipient block to form a TMA block. Two replicated tissue points were sampled from each patient with HCC. Then the wax block was cooled at room temperature for $30 \mathrm{~min}$ and placed in $4^{\circ} \mathrm{C}$ for further usage. The cooled wax block was fixed on the auto tissue slicer and continuously sliced into 50 sections. Each section was $4 \mu \mathrm{m}$. Afterwards, the sections were extended in distilled water for $2 \mathrm{~min}$, then directly mounted onto the superforst plus-treated import slide and dried on the section drier for $16 \mathrm{~h}$ at $60^{\circ} \mathrm{C}$.

Immunohistochemical staining of AMACR was performed by using the two-step Envision plus staining technique. Representative $4 \mu \mathrm{m}$ section TMA was de-paraffinised in xylene and rehydrated in graded ethanol. Then the slides were incubated in 1\% hydrogen peroxide for $30 \mathrm{~min}$ to block endogenous peroxidase activity and then rehydrated in distilled water followed by phosphate buffered saline (PBS). Slides were incubated in $0.1 \mathrm{~mol} / \mathrm{L}$ EDTA ( $\mathrm{pH}$ 9.0) with $3 \mathrm{~min}$ for high-pressure antigen retrieval. Afterwards, the liver sections were incubated with the primary antibody (mouse polyclonal antihuman AMACR antibody, $1: 100$ dilutions, abcam) at $4^{\circ} \mathrm{C}$ overnight. After several rinses with PBS, it was followed by incubating with HRP-conjugated secondary antibodies (ZSGB-BIO, China) at room temperature for another $1 \mathrm{~h}$. Finally, the liver sections were subjected to diaminobenzidine colouration and haematoxylin restaining. The results were independently assessed by two pathologists double-blindly. Using localised prostate cancer as the positive control (scored 3), ${ }^{5}$ all tissues were manually scored as 0 (negative), 1 (weak), 2 (strong) or 3 (very strong). The expression was finally considered by low expression if the final score was $0-1$, while high expression if the final score was 2-3.

\section{Statistical analysis}

All statistical analyses were performed with SPSS software package (V.19.0). Comparisons of quantitative data between two groups were performed with a two-tailed paired Student's test. Categorical data were analysed by Fisher's exact test between
A

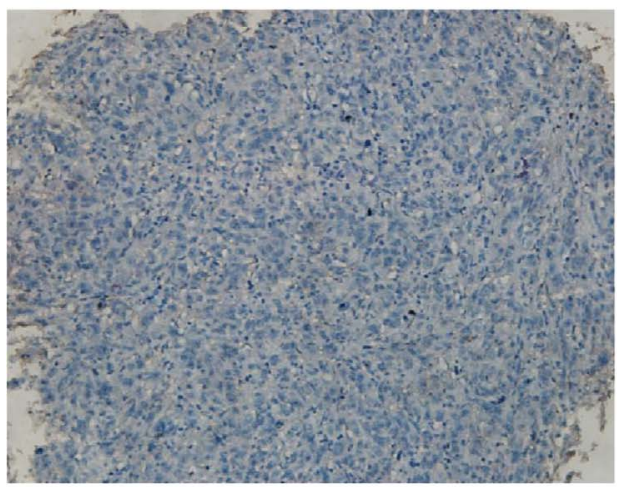

C

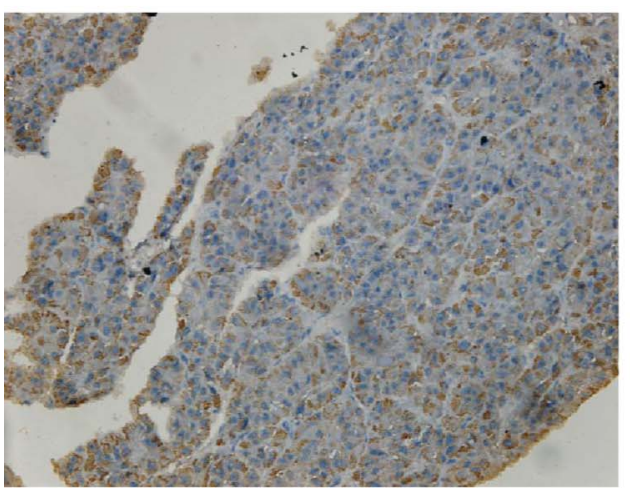

B

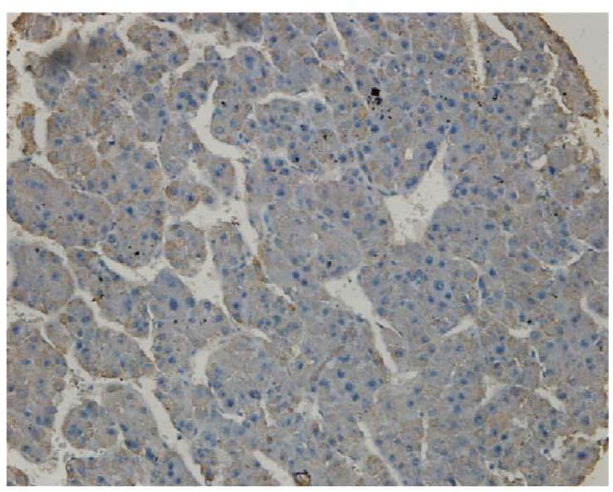

D

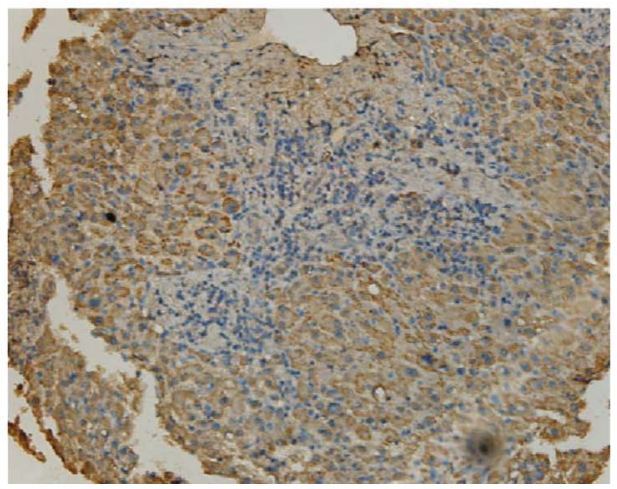

Figure 1 Different $\alpha$-methylacyl-CoA racemase (AMACR) expression levels in hepatocellular carcinoma tumour tissues. As described in 'Methods' section, AMACR were semiquantitatively categorised into four groups: $(A)$ negative $(0)$, (B) weak $(1+),(C)$ moderate $(2+)$ and (D) strong $(3+)$. (original magnifications 200x). 
AMACR expression and clinical-pathological features. Survival curves were calculated by the Kaplan-Meier method and compared using the log-rank test. A Cox proportional hazards model was used to determine the independent factors of survival and recurrence based on the variables selected in univariate analysis. $\mathrm{p}<0.05$ was considered as statistically significant.

\section{RESULTS}

In our previous proteomics study, AMACR was identified to be significantly downregulated in the HCC tumour with early recurrence/metastasis. ${ }^{12}$ Since this protein has clear lower expression profiles in the early recurrence/metastasis patients, it might be interesting potential prognostic biomarker for the predicting of early recurrences/metastases in HCC.

To further confirm the expression profile of AMACR in patients with HCC, we constructed the TMAs and performed the IHC staining for AMACR in a large scale. After independently evaluated by two pathologists, the expression level of AMACR was scored as 0 (negative), 1 (weak), 2 (strong) or 3 (very strong). The representative images of each score are shown in figure 1. Depending on the scores, we divided the AMACR expression level into two groups: low-expression group if the final score was 0 or 1 , and high-expression group if the final score was 2 or 3 . Combining with the clinical history, the major factors that affect the prognosis have been collected to evaluate the relationship between the AMACR expression and the key clinical parameters.

As shown in tables 1 and 2, the low expression of AMACR in HCC tissues was significantly associated with multiple tumour numbers, absence of tumour capsule, undistinguishable tumour

Table 1 Association between $\alpha$-methylacyl-CoA racemase (AMACR) and preoperative clinic features

\begin{tabular}{|c|c|c|c|}
\hline & \multicolumn{2}{|l|}{ AMACR expression } & \multirow[b]{2}{*}{ p Value } \\
\hline & Low expression & High expression & \\
\hline \multicolumn{4}{|l|}{ Gender } \\
\hline Female & $14(67 \%)$ & $7(33 \%)$ & \\
\hline Male & $81(59 \%)$ & $56(41 \%)$ & NS \\
\hline \multicolumn{4}{|l|}{ Age } \\
\hline$<55$ & $61(60 \%)$ & $41(40 \%)$ & \\
\hline$\geq 55$ & $34(61 \%)$ & $22(39 \%)$ & NS \\
\hline \multicolumn{4}{|l|}{$\alpha$-fetoprotein (AFP) ( $\mu \mathrm{g} / \mathrm{L})$} \\
\hline$<1000$ & $51(52 \%)$ & $47(48 \%)$ & \\
\hline$\geq 1000$ & $44(73 \%)$ & $16(27 \%)$ & $0.012^{*}$ \\
\hline \multicolumn{4}{|l|}{ HBV-DNA } \\
\hline Positive & $64(59 \%)$ & $45(41 \%)$ & \\
\hline Negative & $31(63 \%)$ & $18(37 \%)$ & NS \\
\hline \multicolumn{4}{|l|}{ Tumour size $(\mathrm{cm})$} \\
\hline$<5$ & $10(45 \%)$ & $12(55 \%)$ & \\
\hline$\geq 5$ & $85(63 \%)$ & $51(37 \%)$ & NS \\
\hline \multicolumn{4}{|l|}{ No. of tumours } \\
\hline Solitary & $78(56 \%)$ & $61(44 \%)$ & \\
\hline Multiple & $17(89 \%)$ & $2(11 \%)$ & $0.005^{* *}$ \\
\hline Total bilirubin $(\mu \mathrm{mol} / \mathrm{L})$ & $19.411 \pm 25.269$ & $16.997 \pm 6.777$ & NS \\
\hline Albumin ( $g / L)$ & $39.155 \pm 3.977$ & $40.198 \pm 3.977$ & NS \\
\hline Alaninetransaminase (U/L) & $59.033 \pm 47.878$ & $65.984 \pm 67.248$ & NS \\
\hline $\begin{array}{l}\text { Glutamyltranspeptidase } \\
\text { (GGTP) (U/L) }\end{array}$ & $156.733 \pm 167.586$ & $135.311 \pm 106.958$ & NS \\
\hline Prothrombin time (PT) (s) & $13.744 \pm 1.135$ & $13.382 \pm 1.047$ & NS \\
\hline
\end{tabular}

Table 2 Association between the $\alpha$-methylacyl-CoA racemase (AMACR) expression and tumour features

\begin{tabular}{|c|c|c|c|}
\hline & \multicolumn{2}{|c|}{ AMACR expression } & \multirow[b]{2}{*}{ p Value } \\
\hline & Low expression & High expression & \\
\hline \multicolumn{4}{|l|}{ Progression of cirrhosis } \\
\hline Normal/early-stage & $44(57 \%)$ & $33(43 \%)$ & \\
\hline Advanced-stage & $51(63 \%)$ & $30(37 \%)$ & NS \\
\hline \multicolumn{4}{|c|}{ Dissemination to regional lymph nodes } \\
\hline Yes & $7(100 \%)$ & $0(0 \%)$ & \\
\hline No & $88(58 \%)$ & $63(42 \%)$ & $0.042^{*}$ \\
\hline \multicolumn{4}{|l|}{ Tumour capsule } \\
\hline Present & $64(55 \%)$ & $53(45 \%)$ & \\
\hline Absent & $31(76 \%)$ & $10(24 \%)$ & $0.026^{*}$ \\
\hline \multicolumn{4}{|l|}{ Tumour boundaries } \\
\hline Distinct & $83(58 \%)$ & $60(42 \%)$ & \\
\hline Indistinct & $12(86 \%)$ & $2(14 \%)$ & $0.049^{*}$ \\
\hline \multicolumn{4}{|c|}{ Portal vein tumour thrombosis(PVTT) } \\
\hline Yes & $33(79 \%)$ & $9(21 \%)$ & \\
\hline No & $62(53 \%)$ & $54(47 \%)$ & $0.006^{* *}$ \\
\hline \multicolumn{4}{|l|}{ Intraoperative ascites } \\
\hline Yes & $25(70 \%)$ & $11(30 \%)$ & \\
\hline No & 70 (57\%) & $52(43 \%)$ & NS \\
\hline
\end{tabular}

boundaries and presence of portal vein tumour thrombosis (PVTT). Larger tumour size and faster progression of cirrhosis were also more frequently observed in the AMACR lowexpression group, although not statistically significantly. Tumour without encapsulation is more likely to be associated with microscopic vascular invasion ${ }^{13}$ and leads to a further intrahepatic recurrence or metastasis. Meanwhile, it has been reported that tumour recurrence commonly occurs in HCC patients with PVTT, ${ }^{14-17}$ which is the major cause of unsatisfactory prognosis. As shown in table 2,79\% (33/42) of total cases showed AMACR low expression in the PVTT group, and all the cases in the regional lymph nodes dissemination group had a lower AMACR expression. These data suggest that the lower expression of AMACR is well associated with the more invasive features of HCC.

Table 3 Association between the $\alpha$-methylacyl-CoA racemase (AMACR) expression and the postoperative clinical features

\begin{tabular}{|c|c|c|c|}
\hline & \multicolumn{2}{|c|}{ AMACR expression } & \multirow[b]{2}{*}{$\begin{array}{l}\mathbf{p} \\
\text { Value }\end{array}$} \\
\hline & $\begin{array}{l}\text { Low } \\
\text { expression }\end{array}$ & $\begin{array}{l}\text { High } \\
\text { expression }\end{array}$ & \\
\hline \multicolumn{4}{|l|}{ Recurring type } \\
\hline Regional recurring & $18(67 \%)$ & $9(33 \%)$ & \\
\hline Distant recurring & $57(63 \%)$ & $33(37 \%)$ & NS \\
\hline \multicolumn{4}{|l|}{ Histological grade } \\
\hline Well differentiated (I) & $10(50 \%)$ & $10(50 \%)$ & \\
\hline Moderately differentiated (II) & $59(61 \%)$ & $38(39 \%)$ & \\
\hline Poorly differentiated (III) & $26(63 \%)$ & $15(37 \%)$ & NS \\
\hline \multicolumn{4}{|l|}{ Extrahepatic metastasis } \\
\hline Yes & $21(75 \%)$ & $7(25 \%)$ & \\
\hline No & $74(57 \%)$ & $56(43 \%)$ & NS \\
\hline
\end{tabular}


Table 4 The expression of $\alpha$-methylacyl-CoA racemase (AMACR) in different groups according to the time of recurrence/metastasis postoperation

\begin{tabular}{|c|c|c|c|c|c|}
\hline \multirow[b]{2}{*}{ Groups } & \multicolumn{4}{|c|}{ AMACR expression } & \multirow[b]{2}{*}{$p$ Values } \\
\hline & Numbers & Low & High & $\begin{array}{l}\text { Low expression } \\
\text { rate }(\%)\end{array}$ & \\
\hline $\mathrm{R} / \mathrm{M}_{\leq 12 \text { months }}$ group & 101 & 71 & 30 & 70.3 & $<0.001$ * * \\
\hline $\mathrm{R} / \mathrm{M}_{12-24 \text { months group }}$ & 21 & 12 & 9 & 57.1 & 0.1 \\
\hline NR/M group & 36 & 12 & 24 & 33.3 & \\
\hline
\end{tabular}

The associations between the expression level of AMACR and postoperative clinical features have been further studied and shown in table 3 . The AMACR expression has been assessed in 158 patients, including 101 cases of early recurrence/ metastasis ( $\mathrm{R} / \mathrm{M}_{\leq 12}$ months $), 21$ cases of late recurrence/metastasis $\left(\mathrm{R} / \mathrm{M}_{12-24}\right.$ months $)$ and 36 cases of none-recurrence/metastasis $(\mathrm{NR} / \mathrm{M})$. As shown in table 4, the low expression of AMACR was detected in 12 cases of NR/M HCC patients (in a total of 36 patients, the low-expression rate was $33.3 \%$ ). In contrast, the AMACR low-expression rate was significantly higher in the early recurrence/metastasis group $\left(\mathrm{R} / \mathrm{M}_{\leq 12}\right.$ months, 71 of 101 , low-expression rate $70.3 \%, \mathrm{p}<0.001)$. Meanwhile, the patients with low expression of AMACR had very discouraging prognosis, only with a median survival of 17 months; however, the patients with high expression of AMACR had a better prognosis, with a median survival of 45 months (figure 2).

The univariate and multivariate analysis was further performed to evaluate the prognosis potential of AMACR, as shown in table 5; low expression of AMACR was the independent risk factor affecting the survival of patients with HCC. These data

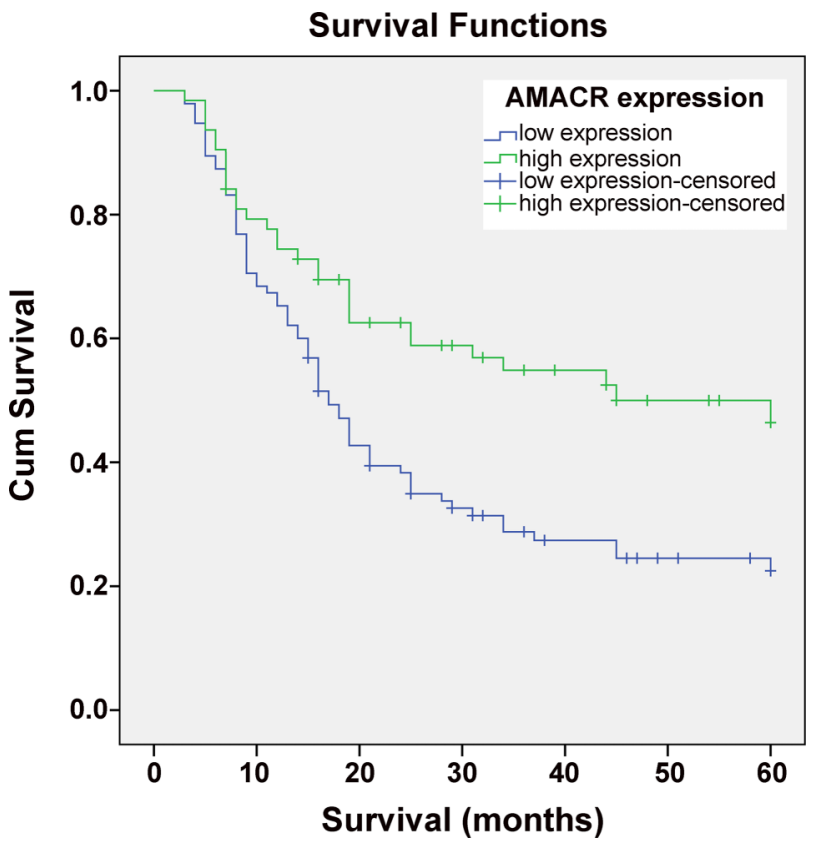

Figure 2 Kaplan-Meier's analysis of the correlation between the $\alpha$-methylacyl-CoA racemase (AMACR) expression and the survival of hepatocellular carcinoma (HCC) patients. Patients with HCC with low-expression level of AMACR $(n=95)$ had significantly lower survival rate than those with high-expression level of AMACR $(n=63)$, $(p<0.001)$. clearly suggest that AMACR could serve as an independent prognostic factor for the early recurrence/metastasis of HCC.

\section{DISCUSSION}

Following curative liver resection of HCC within the Milan criteria, still up to $63 \%$ of patients will develop intrahepatic recurrence or extrahepatic metastasis within 5 years, ${ }^{18}$ and most of the recurrence/metastasis will occur in the early stage after the surgery. The incidence of recurrence/metastasis within 12 months in the residual liver of patients with HCC, who underwent curative resection, ranges from $50 \%$ to $70 \%{ }^{19-21}$; and the median survival after recurrence/metastasis is only 13 months. ${ }^{22}$ There are several biomarkers for predicting the recurrence/metastasis of HCC, but still limited biomarkers point to the early stage. Here, the prognostic potential of AMACR for the prediction of early recurrence/metastasis postliver resection in HCC has been systematically analysed.

Our previous studies have shown that the AMACR was significantly downregulated in the early recurrence/metastasis group compared with the NR/M group. ${ }^{12}$ These results encouraged us to further verify the expression profiles of AMACR in patients with HCC in a large scale; therefore, we performed the TMA with immunohistochemistry (IHC) staining to evaluate the AMACR expression in patients.

Combining with the clinical assessment, several important tumour features have been analysed. Among those features, the preoperative serum $\alpha$-fetoprotein level, tumour numbers, dissemination to regional lymph nodes, tumour capsule and PVTT are significantly correlated with the low expression of AMACR. Additionally, patients with HCC with lower AMACR expression are significantly associated with a poorer 5 -year survival with a median survival of 17 months, but the patients with HCC with higher AMACR expression had a better survival with a median survival of 45 months. Furthermore, Cox's multivariate proportional hazards model revealed that the AMACR expression level can serve as an independent prognostic biomarker to identify patients with poor clinical outcomes. Taken together, these data suggested that AMACR can be applied as a potential prognosis biomarker for the recurrence/metastasis of HCC in patients. Further validation of the AMACR expression by RT-PCR on mRNA level is not necessary for the clinical practice, but it would certainly promote the clinical applications of AMACR due to the easier use of the RT-PCR technique. As a promising prognosis biomarker, it would be crucial to detect in the circulation since the biopsies are not always available in clinical practice; we have some unpublished preliminary data showing that the AMACR could be detected in HCC patient's serum, but further study is needed to clarify whether the AMACR serum levels are associated with the prognosis of HCC, as well as its sensitivity and specificity.

It has been reported that lower AMACR expression was also associated with worse outcome in the prostate cancer. ${ }^{23}$ Meanwhile, the AMACR downregulation in the prostatic carcinoma metastases ${ }^{5}{ }^{24}$ has also been identified; similar findings also have been reported in colorectal carcinoma ${ }^{25}$ and in adenocarcinomas of the small and large intestines as well. ${ }^{26}$ However, on the other hand, the AMACR overexpression has also been identified in various cancers. ${ }^{27}$ As described previously, the AMACR regulates the entry of branched-chain lipids into the peroxisomal and mitochondrial $\beta$-oxidation pathways, and then regulates the production of reactive oxygen species, which could result in DNA damage due to oxidative stress. ${ }^{28}$ It seems that the upregulation of AMACR may be involved in the early tumourigenesis, but the downregulation might relate to the development of tumours with high capacity of recurrence/metastasis. Although 
Table 5 Univariate and multivariate analysis of $\alpha$-methylacyl-CoA racemase (AMACR) and other clinicopathological characteristics at 5 years of follow-up

\begin{tabular}{|c|c|c|c|c|c|c|}
\hline \multirow[b]{3}{*}{ Variables } & \multicolumn{6}{|c|}{ Survival } \\
\hline & \multicolumn{3}{|c|}{ Univariate analysis } & \multicolumn{3}{|c|}{ Multivariate analysis } \\
\hline & HR & $95 \% \mathrm{Cl}$ & p Value & HR & $95 \% \mathrm{Cl}$ & p Value \\
\hline Age $(<55 / \geq 55)$ & 0.743 & 0.488 to 1.132 & 0.167 & & & \\
\hline Sex (male/female) & 2.865 & 1.327 to 60185 & 0.007 & 2.620 & 1.200 to 5.722 & 0.016 \\
\hline HBV infection (absent/present) & 1.411 & 0.929 to 2.142 & 0.106 & & & \\
\hline Cirrhosis (absent/present) & 1.054 & 0.770 to 1.442 & 0.744 & & & \\
\hline Alanine transaminase (ALT) $(\mathrm{U} / \mathrm{L})(<54 / \geq 54)$ & 1.167 & 0.781 to 1.745 & 0.451 & & & \\
\hline Total bilirubin $(\mu \mathrm{mol} / \mathrm{L})(<25 / \geq 25)$ & 0.765 & 0.386 to 1.519 & 0.445 & & & \\
\hline GGTP (U/L) $(<60 / \geq 60)$ & 1.634 & 0.991 to 2.696 & 0.054 & & & \\
\hline Prothrombin time $(s)(\leq 14 />14)$ & 0.985 & 0.626 to 1.549 & 0.946 & & & \\
\hline Dissemination to regional lymph nodes (absent/present) & 2.280 & 0.993 to 5.234 & 0.052 & & & \\
\hline Tumour boundaries (distinct/indistinct) & 1.417 & 0.737 to 2.723 & 0.296 & & & \\
\hline Intraoperative ascites (absent/present) & 1.628 & 1.050 to 2.524 & 0.030 & 1.251 & 0.776 to 2.015 & 0.358 \\
\hline Tumour number (single/multiple) & 0.992 & 0.530 to 1.857 & 0.980 & & & \\
\hline Maximal tumour size $(<5 / \geq 5)$ & 4.439 & 1.084 to 10.925 & 0.001 & 2.368 & 0.931 to 6.026 & 0.070 \\
\hline Tumour encapsulation (absent/present) & 1.833 & 1.203 to 2.795 & 0.005 & 1.301 & 0.822 to 2.058 & 0.262 \\
\hline Tumour differentiation (I/II-III) & 0.258 & 0.105 to 0.635 & 0.003 & 0.418 & 0.167 to 1.050 & 0.063 \\
\hline Extrahepatic metastasis (yes/no) & 0.851 & 0.505 to 1.434 & 0.544 & & & \\
\hline PVTT (absent/present) & 2.280 & 1.505 to 30454 & 0.000 & 1.801 & 1.169 to 2.773 & 0.008 \\
\hline AMACR (low expression/high expression) & 0.501 & 0.326 to 0.770 & 0.002 & 0.612 & 0.319 to 0.957 & 0.031 \\
\hline
\end{tabular}

there are some mechanistic studies on AMACR, ${ }^{29} 30$ the underlying molecular mechanisms that explain the aggressive behaviour and early recurrence/metastasis feature of HCC with lower AMACR expression are still unclear. Further prospective studies with molecular mechanism are necessary to fully clarify the clinical implications of AMACR in HCC.

In conclusion, we applied TMA-based immunohistochemistry staining to perform a large-scale cohort clinical study and showed that the low expression of AMACR was significantly associated with key clinical features and hence leads to the early recurrence/metastasis of HCC. Our findings indicate that the AMACR might be a potential prognostic biomarker for predicting the early recurrences/metastases of HCC after hepatectomy.

\section{Take home messages}

- $\alpha$-Methylacyl-CoA racemase (AMACR) is downregulated in the early recurrence/metastasis hepatocellular carcinoma (HCC) tumour tissues.

- The downregulation of AMACR is significantly associated with poor prognosis of patients with HCC.

- AMACR might be a promising prognostic biomarker for patients with HCC instead of a diagnostic biomarker.

Contributors $B X, Y Z, Z C$ and $L C$ performed the experiments; $B X$ and $X D$ collected the clinical samples; $\mathrm{BX}$ and $\mathrm{XL}$ wrote the paper; $\mathrm{XL}, \mathrm{AH}$ and $\mathrm{JL}$ designed the project.

Funding This work was supported by the key project of the National Science and Technology of China (grant no. 2012ZX10002010-001-006 and grant no.
2012ZX10002016-013), the National Natural Science Foundation of China (grant no. 31201008), the Backbone Talents Training Project of Fujian Health Department (grant no. 2013-ZQN-ZD-29), the key project of Fujian Science and Technology Department (grant no. 2014Y0027), the Scientific Foundation of Fuzhou Health Department (grant no. 2013-s-wp1 and grant no. 2013-s-w12) and the research development foundation of Fujian Medical University (grant no. FZS13001Y and grant no. FZS13005Z).

\section{Competing interests None.}

Patient consent Obtained.

Ethics approval The Institution Review Board of the First Affiliated Hospital of Fujian Medical University.

Provenance and peer review Not commissioned; externally peer reviewed.

Open Access This is an Open Access article distributed in accordance with the Creative Commons Attribution Non Commercial (CC BY-NC 4.0) license, which permits others to distribute, remix, adapt, build upon this work non-commercially, and license their derivative works on different terms, provided the original work is properly cited and the use is non-commercial. See: http://creativecommons.org/ licenses/by-nc/4.0/

\section{REFERENCES}

1 Chen WQ, Zeng HM, Zheng RS, et al. Cancer incidence and mortality in china, 2007. Chin J Cancer Res 2012;24:1-8.

2 Chen XP, Qiu FZ, Wu ZD, et al. Hepatectomy for huge hepatocellular carcinoma in 634 cases. World I Gastroenterol 2006;12:4652-5.

3 Liau KH, Ruo L, Shia J, et al. Outcome of partial hepatectomy for large $(>10 \mathrm{~cm})$ hepatocellular carcinoma. Cancer 2005:104:1948-55.

4 Ferdinandusse $\mathrm{S}$, Denis $\mathrm{S}$, IJ L, et al. Subcellular localization and physiological role of alpha-methylacyl-CoA racemase. Journal of lipid research 2000;41:1890-6.

5 Rubin MA, Zhou M, Dhanasekaran SM, et al. alpha-Methylacyl coenzyme A racemase as a tissue biomarker for prostate cancer. JAMA 2002;287:1662-70.

6 Luo J, Zha S, Gage WR, et al. Alpha-methylacyl-CoA racemase: a new molecular marker for prostate cancer. Cancer Res 2002;62:2220-6.

$7 \mathrm{Xu}$ J, Stolk JA, Zhang $X$, et al. Identification of differentially expressed genes in human prostate cancer using subtraction and microarray. Cancer Res 2000;60:1677-82. 
8 Evans AJ. Alpha-methylacyl CoA racemase (P504S): overview and potential uses in diagnostic pathology as applied to prostate needle biopsies. J Clin Pathol 2003;56:892-7.

9 Guzman G, Wu SJ, Kajdacsy-Balla A, et al. Alpha-methylacyl-CoA racemase (AMACR/P504S) can distinguish hepatocellular carcinoma and dysplastic hepatocytes from benign nondysplastic hepatocytes. Appl Immunohistochem Mol Morphol 2006;14:411-16.

10 Li W, Cagle PT, Botero RC, et al. Significance of overexpression of alpha methylacyl-coenzyme A racemase in hepatocellular carcinoma. J Exp Clin Cancer Res 2008:27:2.

11 Willemoe GL, Vainer B. AMACR is not applicable as a diagnostic tool in hepatocellular carcinoma. APMIS 2010;118:85-90.

12 Huang $X$, Zeng $Y$, Xing $X$, et al. Quantitative proteomics analysis of early recurrence/metastasis of huge hepatocellular carcinoma following radical resection. Proteome Sci 2014;12:22.

13 Kim BK, Han KH, Park YN, et al. Prediction of microvascular invasion before curative resection of hepatocellular carcinoma. J Surg Oncol 2008;97:246-52.

14 Ohkubo T, Yamamoto J, Sugawara Y, et al. Surgical results for hepatocellular carcinoma with macroscopic portal vein tumor thrombosis. J Am Coll Surg 2000;191:657-60.

15 Chen XP, Qiu FZ, Wu ZD, et al. Effects of location and extension of portal vein tumor thrombus on long-term outcomes of surgical treatment for hepatocellular carcinoma. Ann Surg Oncol 2006;13:940-6.

16 Kumada K, Ozawa K, Okamoto R, et al. Hepatic resection for advanced hepatocellular carcinoma with removal of portal vein tumor thrombi. Surgery 1990;108:821-7.

17 Fukuda S, Okuda K, Imamura $M$, et al. Surgical resection combined with chemotherapy for advanced hepatocellular carcinoma with tumor thrombus: report of 19 cases. Surgery 2002;131:300-10.

18 Lim KC, Chow PK, Allen JC, et al. Systematic review of outcomes of liver resection for early hepatocellular carcinoma within the Milan criteria. Br J Surg 2012;99:1622-9.
19 Choi GH, Han DH, Kim DH, et al. Outcome after curative resection for a huge (>0r=10 cm) hepatocellular carcinoma and prognostic significance of gross tumor classification. Am J Surg 2009;198:693-701.

20 Shah SA, Wei AC, Cleary SP, et al. Prognosis and results after resection of very large $(>0 \mathrm{r}=10 \mathrm{~cm})$ hepatocellular carcinoma. J Gastrointest Surg 2007:11:589-95.

$21 \mathrm{Ng} \mathrm{KM}$, Yan TD, Black D, et al. Prognostic determinants for survival after resection/ ablation of a large hepatocellular carcinoma. HPB 2009;11:311-20.

22 Lee SG, Hwang S, Jung JP, et al. Outcome of patients with huge hepatocellular carcinoma after primary resection and treatment of recurrent lesions. Br J Surg 2007;94:320-6.

23 Rubin MA, Bismar TA, Andren 0, et al. Decreased alpha-methylacyl CoA racemase expression in localized prostate cancer is associated with an increased rate of biochemical recurrence and cancer-specific death. Cancer Epidemiol Biomarkers Prev 2005;14:1424-32.

24 Rubin MA, Zerkowski MP, Camp RL, et al. Quantitative determination of expression of the prostate cancer protein alpha-methylacyl-CoA racemase using automated quantitative analysis (AQUA): a novel paradigm for automated and continuous biomarker measurements. Am J Pathol 2004;164:831-40.

25 Lin A, Weiser MR, Klimstra DS, et al. Differential expression of alpha-methylacyl-coenzyme A racemase in colorectal carcinoma bears clinical and pathologic significance. Hum Pathol 2007;38:850-6

26 Chen ZM, Ritter JH, Wang HL. Differential expression of alpha-methylacyl coenzyme A racemase in adenocarcinomas of the small and large intestines. Am J Surg Pathol 2005;29:890-6

27 Jiang Z, Woda BA, Rock KL, et al. P504S: a new molecular marker for the detection of prostate carcinoma. Am J Surg Pathol 2001;25:1397-404.

28 Schrader M, Fahimi HD. Peroxisomes and oxidative stress. Biochim Biophys Acta 2006;1763:1755-66.

29 Lloyd MD, Darley DJ, Wierzbicki AS, et al. Alpha-methylacyl-CoA racemase-an 'obscure' metabolic enzyme takes centre stage. FEBS J 2008;275:1089-102.

30 Sekine S, Ogawa R, Ojima $H$, et al. Overexpression of alpha-methylacyl-CoA racemase is associated with CTNNB1 mutations in hepatocellular carcinomas. Histopathology 2011;58:712-19. 